\title{
39
}

\section{Re-initialization in discontinuous systems}

\author{
J.M. Schumacher
}

CWI

P. O. Box 94079

1090 GB Amsterdam

THE NETHERLANDS

Hans.Schumacher@cwi.nI

\section{Description of the problem}

Let an implicit system of differential equations be given in the form

$$
f(x(t), \dot{x}(t))=0
$$

where $x$ is an $n$-dimensional vector and $f$ is a smooth mapping from $\mathbb{R}^{2 n}$ to $\mathbb{R}^{n}$. More generally $x$ may take values in a differentiable manifold and $f$ may be defined on the tangent bundle. Let us first consider the case that $f$ is linear, so that the equation (39.1) may be written as

$$
E \dot{x}(t)=F x(t)
$$

where both $E$ and $F$ are matrices of size $n \times n$. To the pair of matrices $(E, F)$ one may associate two subspaces of $\mathbb{R}^{n}$, in the following way. Denote by $V(E, F)$ the limit of the sequence defined by

$$
V^{0}=\mathbb{R}^{n}, \quad V^{j+1}=F^{-1} E V^{j} \quad(j=0,1, \ldots) .
$$

Alternatively, $V(E, F)$ may be defined as the largest element of the set of all subspaces $V$ that satisfy $F V \subset E V$. Secondly, denote by $T(E, F)$ the limit of the sequence defined by

$$
T^{0}=\{0\}, \quad T^{j+1}=E^{-1} F T^{j} \quad(j=0,1, \ldots) .
$$

It is equivalent to say that $T(E, F)$ is the smallest element of the set of all subspaces $T$ such that $T \supset E^{-1} F T$. We can now state the following well-known theorem.

Theorem 1 For a pair of square real matrices $(E, F)$, the following statements are equivalent: 
(i) the polynomial $\operatorname{det}(s E-F)$ is nonzero;

(ii) the subspaces $V(E, F)$ and $T(E, F)$ form a direct sum decomposition of $\mathbb{R}^{n}$;

(iii) the differential equation (39.2) is well-posed in the sense that for each $x_{0} \in \mathbb{R}^{n}$ there is at most one solution of (39.2) passing through $x_{0}$.

Actually more precise statements could be made; in particular the "consistent subspace", that is, the space of all $x_{0}$ through which a smooth solution of (39.2) passes, is equal to $V(E, F)$, and the subspace $T(E, F)$ has an interpretation in terms of impulsive solutions to $(39.2)[14,11]$.

The theorem connects statements of three different types: an algebraic one, a geometric one, and a dynamic one (relating to differential equations). The question that we are interested in is now the following: to what extent do there exist nonlinear generalizations of Thm. 1 ? So, for a nonlinear implicit system of the form (39.1), we would like to know whether well-posedness can be connected to an algebraic criterion as in (i) above, and in particular (as motivated below) under what conditions there is an associated state space decomposition similar to (ii).

In the nonlinear case as given by (39.1) we can still define the consistent manifold as the set of all points $x$ through which a smooth solution of (39.1) passes. Let us also define the constraint manifold as the set of all $x$ for which there is a $v$ such that $f(x, v)=0$. Note that, in the linear case, the constraint manifold is exactly the subspace $V^{1}$ in the sequence defined by (39.3).

\section{Motivation of the problem}

Consider a linear system with scalar inputs and outputs

$$
\begin{aligned}
\dot{x}(t) & =A x(t)+B u(t) \\
y(t) & =C x(t)
\end{aligned}
$$

together with inequality constraints of the following form:

$$
\text { for all } t: \quad y(t) \geq 0, \quad u(t) \geq 0, \text { and } y(t)=0 \text { or } u(t)=0 .
$$

These could for instance be the equations of a linear electrical network with an ideal diode; also, equations of the same form arise from an application of Pontryagin's maximum principle to a linear-quadratic problem with a single linear inequality constraint. In the situation in which the constraint is active $(y=0)$, the system dynamics is given by

$$
\frac{d}{d t}\left[\begin{array}{ll}
I & 0 \\
0 & 0
\end{array}\right]\left[\begin{array}{l}
x \\
u
\end{array}\right](t)=\left[\begin{array}{ll}
A & B \\
C & 0
\end{array}\right]\left[\begin{array}{l}
x \\
u
\end{array}\right](t) .
$$


This is an equation of the form (39.2). If we assume that the system is well-posed, the conditions of the theorem mentioned above hold and so the consistent subspace is $V(E, F)$ with

$$
E=\left[\begin{array}{ll}
I & 0 \\
0 & 0
\end{array}\right], \quad F=\left[\begin{array}{cc}
A & B \\
C & 0
\end{array}\right]
$$

Note that while it is natural to let a change from unconstrained to constrained mode take place only at times $t$ at which both $y(t)$ and $u(t)$ vanish, the ensuing conditions $x \in \operatorname{ker} C$ and $u=0$ may not be enough to guarantee that the vector $\left[\begin{array}{l}x \\ u\end{array}\right]$ is in $V(E, F)$. However we are always able to project a given vector $\left[\begin{array}{l}x \\ u\end{array}\right]$ onto $V(E, F)$ along the complementary subspace $T(E, F)$, and doing so is in fact supported by the interpretation of the latter space as the space of jump directions. The same recipe can be given in the case of systems multiple inputs and outputs connected by "complementarity conditions" of the form (39.7) (taken componentwise). Of course in the multivariable case there are more possible combinations of active and inactive constraints; a system with $k$ constraints has $2^{k}$ modes. In this way it becomes possible to give an unequivocal specification of what is to be understood by a solution of a system of the form (39.5-39.7). The dynamical systems that are obtained in this way have been called linear complementarity systems [15]. Surely, to define the concept of a solution is one thing and to prove existence and uniqueness of solutions is another; sufficient conditions for well-posedness in terms of the classical linear input-output system (39.5) are given in [15].

Another example of a class of discontinuous dynamical systems for which a complete specification of the dynamics can be given is provided by mechanical systems subject to inequality constraints on the configuration variables; here the projection rule as defined by Moreau [25] can be used for reinitialization. In the class of general nonlinear systems, the re-initialization problem becomes vacuous if the consistent manifold coincides with the constraint manifold, so that the constraint only become active in situations in which the state is already in the consistent manifold corresponding to the relevant constraints, and state trajectories can be required to be continuous. Otherwise however there is a nontrivial re-initialization problem to solve, which will require a decomposition of the constraint manifold into the consistent manifold and an associated foliation - that is, a decomposition similar to the one in the theorem above.

\section{History and related results}

Theorem 1 above goes back 'essentially' to the 19th century, at least as far as the equivalence between parts (i) and (ii) is concerned, since the implication from (i) to (ii) can be read off from the Weierstrass canonical 
form for regular matrix pencils [32], and the implication from (ii) to (i) follows from the canonical form for singular matrix pencils given by Kronecker [20]. Weierstrass and Kronecker did not explicitly use the algorithms (39.3) and (39.4); these occur perhaps for the first time in a paper of 1946 by Dieudonné [6]. Gantmacher [9] presented a derivation of the Kronecker canonical form that is much simplified with respect to the original version, and also pointed out the implications of the canonical form for systems of implicit linear differential equations with constant coefficients. For systems in the special form (39.9) the algorithms (39.3) and (39.4) take on a special form as well, under which they have become well-known in control theory through the work of in particular Wonham [33], Morse [26], and Basile and Marro [1].

Implicit systems of nonlinear equations occur naturally in the modeling of physical phenomena, and in recent years there has been great interest in the development of numerical methods for solving such systems, see for instance $[2,12]$. Implicit systems are categorized in classes of increasing difficulty of numerical solution by a quantity known as the "index". There are actually various definitions of this notion (cf. [5] for a discussion of several proposals), but roughly speaking the index is related to the number of "hidden equations" that are not explicitly formulated in the model equations but that follow from them by differentiation. The index can also be seen as a measure of the difference between the dimension of the consistent manifold and the dimension of the constraint manifold, so that problems that cause numerical difficulties are also problems that cause difficulties with re-initialization. One may surmise that this connection is not accidental; indeed the index reduction scheme proposed by Gear [10] defines a re-initialization mapping at least in a neighborhood of the consistent manifold, cf. the remark at the end of the cited paper.

It would appear therefore that the large literature on the index of implicit nonlinear systems will be of relevance to the re-initialization problem. Unfortunately this literature gives mixed clues; as already noted there are several definitions of the index which can give widely different results [5]. A case can be made for the claim that a study of the index should be based on methods from differential algebra. Steps in this direction have been taken by Fliess and co-authors [8,7]. Prolongations (corresponding to 'repeated differentiation') have been used by Fliess et al. as well, and they have obtained a nonlinear version of the equivalence between (i) and (iii) in terms of differential functional independence [7]. See also the work by Le Vey [22] who develops connections to the formal theory of partial differential equations. The prolongation method is related to the use of 'dummy derivatives' in numerical treatments of implicit systems [24]. As an alternative to prolongation one may attempt to use more geometrically oriented methods as would be suggested by the linear version (39.4). A nonlinear variant of (39.4) has been proposed by Isidori et al. [18] although not at the level of generality of (39.1); moreover in some examples the algorithm of 
[18] does not quite give the desired results, see [30, Remark 5.8]. Nonlinear versions of the algorithm (39.3) are well-known [17, 27, 28].

The linear case also suggests a connection to what is known in linear system theory as the "structure at infinity" (see for instance [21]). Indeed, the index of the linear system (39.2) can be defined as one plus the order of the pole at infinity of the rational matrix function $(s E-F)^{-1}$. Actually there are in general several poles at infinity, as indicated by the Smith-MacMillan form at infinity $[13,19]$, and this suggests that the index should be defined as a vector rather than as a single number. It has been shown by Bujakiewicz [4] that this extension is actually important for numerical purposes. Above it has been noted already that the subspace $T(E, F)$ has an interpretation in terms of impulsive solutions of the equation (39.2), which then needs to be interpreted in a generalized sense. This suggests that 'solving' (39.1) with an inconsistent initial condition (which is a possible interpretation of the re-initialization problem) will bring in the theory of impulsive solutions of nonlinear differential equations, which is a subject of its own. It may be expected that certain commutativity conditions on vector fields will be needed to ensure that solutions are well-defined; this is in line with the fact that involutivity conditions are often found to play an important role in applications of the nonlinear variant of (39.4) as given in [18], see for instance [31]. If such conditions are not satisfied it may happen that the re-initialization problem is not well-posed and one will either have to reformulate the model or accept a certain degree of indeterminism.

\section{References}

[1] G. Basile and G. Marro. Controlled and Conditioned Invariants in Linear System Theory. Prentice Hall, Englewood Cliffs, NJ, 1992.

[2] K. E. Brenan, S. L. Campbell, and L. R. Petzold. Numerical Solution of Initial-Value Problems in Differential-Algebraic Equations. NorthHolland, Amsterdam, 1989.

[3] B. Brogliato. Nonsmooth Impact Mechanics. Models, Dynamics and Control. Lect. Notes Contr. Inform. Sci. 220. Springer, Berlin, 1996.

[4] P. Bujakiewicz. Maximum weighted matching for high index differential algebraic equations. $\mathrm{PhD}$ thesis, TU Delft, 1994.

[5] S.L. Campbell and C.W. Gear. The index of general nonlinear DAEs. Numer. Math., 72:173-196, 1995.

[6] J. Dieudonné. Sur la réduction canonique des couples des matrices. Bull. Soc. Math. France, 74:130-146, 1946.

[7] M. Fliess, J. Lévine, Ph. Martin, and P. Rouchon. Index and decomposition of nonlinear implicit differential equations. In Proc. IFAC 
Conference on System Structure and Control (Nantes, France, July 1995), pages 43-48, 1995.

[8] M. Fliess, J. Lévine, and P. Rouchon. Index of a general differentialalgebraic implicit system. In H. Kimura and S. Kodama, editors, Recent Advances in Mathematical Theory of Systems, Control, Networks and Signal Processing (Proc. Int. Symp. MTNS-91, Kobe, Japan, June 1991), pages 289-294. Mita Press, 1992.

[9] F. R. Gantmacher. The Theory of Matrices (Vol. II). Chelsea, New York, 1959.

[10] C. W. Gear. Differential-algebraic equation index transformations. SIAM J. Sci. Stat. Comput., 9:39-47, 1988.

[11] A. H. W. Geerts and J. M. Schumacher. Impulsive-smooth behavior in multimode systems. Part I: State-space and polynomial representations. Automatica, 32:747-758, 1996.

[12] E. Hairer and G. Wanner. Solving Ordinary Differential Equations II: Stiff and Differential-Algebraic Problems. Springer, Berlin, 1991.

[13] M. L. J. Hautus. The formal Laplace transform for smooth linear systems. In G. Marchesini and S.K. Mitter, editors, Mathematical Systems Theory, Lect. Notes Econ. Math. Syst. 131, pages 29-47. Springer, New York, 1976.

[14] M. L. J. Hautus and L. M. Silverman. System structure and singular control. Lin. Alg. Appl., 50:369-402, 1983.

[15] W. P. M. H. Heemels, J. M. Schumacher, and S. Weiland. Linear complementarity systems. Internal Report 97 I/01, Measurement and Control Systems, Dept. of EE, Eindhoven Univ. of Technol., July 1997. http: //www.cwi.nl/ jms/lcs.ps.Z.

[16] W. P. M. H. Heemels, J. M. Schumacher, and S. Weiland. The rational complementarity problem. Internal Report $98 \mathrm{I} / 02$, Measurement and Control Systems, Dept. of EE, Eindhoven Univ. of Technol., May 1998.

http://www.cwi.nl/ jms/PUB/rcp.ps.Z.

[17] A. Isidori. Nonlinear Control Systems: An Introduction. Lect. Notes Contr. Inf. Sci. Springer, Berlin, 1985.

[18] A. Isidori, A.J. Krener, C. Gori-Giorgi, and S. Monaco. Nonlinear decoupling via feedback: a differential-geometric approach. IEEE Trans. Automat. Contr., AC-26:331-345, 1981. 
[19] T. Kailath. Linear Systems. Prentice-Hall, Englewood Cliffs, N.J., 1980.

[20] L. Kronecker. Algebraische Reduction der Schaaren bilinearer Formen. S.-B. Akad. Berlin, pages 763-776, 1890.

[21] M. Kuijper and J. M. Schumacher. Input-output structure of linear differential/algebraic systems. IEEE Trans. Automat. Contr., AC38:404-414, 1993.

[22] G. Le Vey. Differential algebraic equations, a new look at the index. Rapport de recherche 2239, INRIA, Rennes, 1994.

[23] O. Maler, editor. Hybrid and Real-Time Systems. (Proc. Intern. Workshop HART'97, Grenoble, France, March 1997.) Lect. Notes Comp. Sci. 1201, Berlin, 1997. Springer.

[24] S.E. Mattson and G. Söderlind. A new technique for solving highindex differential-algebraic equations using dummy derivatives. In Proc. 1992 IEEE Symp. on Computer-Aided Control System Design (CACSD '92), Napa, Ca., March 1992, pages 218-224, 1992.

[25] J. J. Moreau. Liaisons unilatérales sans frottement et chocs inélastiques. C. R. Acad. Sc. Paris, 296:1473-1476, 1983.

[26] A. S. Morse. Structural invariants of linear multivariable systems. SIAM J. Control, 11:446-465, 1973.

[27] H. Nijmeijer and A. J. van der Schaft. Nonlinear Dynamical Control Systems. Springer-Verlag, Berlin, 1990.

[28] P.J. Rabier and W.C. Rheinboldt. A geometric treatment of implicit differential-algebraic equations. J. Diff. Eq., 109:110-146, 1994.

[29] A. J. van der Schaft and J. M. Schumacher. Complementarity modeling of hybrid systems. IEEE Trans. Automat. Contr., AC-43:483-490, 1998.

[30] A. J. van der Schaft and J. M. Schumacher. The complementaryslackness class of hybrid systems. Math. Contr. Signals Syst., 9:266301, 1996.

[31] A.J. van der Schaft. On realization of nonlinear systems described by higher-order differential equations. Math. Syst. Th., 19:239-275, 1987. Correction: Math. Syst. Th., 20:305-306, 1987.

[32] K. Weierstrass. Zur Theorie der bilinearen und quadratischen Formen. Monatsh. Akad. Wiss., pages 310-338, 1867.

[33] W. M. Wonham. Linear Multivariable Control: A Geometric Approach. Springer Verlag, Heidelberg, 2nd edition, 1979. 